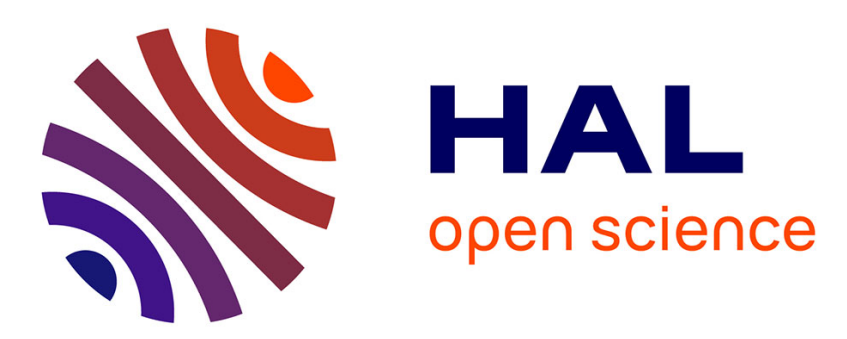

\title{
LOWER BOUNDS FOR THE EIGENVALUES OF THE Spin c DIRAC OPERATOR ON SUBMANIFOLDS
}

\author{
Roger Nakad, Julien Roth
}

\section{To cite this version:}

Roger Nakad, Julien Roth. LOWER BOUNDS FOR THE EIGENVALUES OF THE Spin c DIRAC OPERATOR ON SUBMANIFOLDS. Archiv der Mathematik, 2015, 104 (5), pp.451-461. hal01112320

\section{HAL Id: hal-01112320 \\ https://hal.science/hal-01112320}

Submitted on 2 Feb 2015

HAL is a multi-disciplinary open access archive for the deposit and dissemination of scientific research documents, whether they are published or not. The documents may come from teaching and research institutions in France or abroad, or from public or private research centers.
L'archive ouverte pluridisciplinaire HAL, est destinée au dépôt et à la diffusion de documents scientifiques de niveau recherche, publiés ou non, émanant des établissements d'enseignement et de recherche français ou étrangers, des laboratoires publics ou privés. 


\title{
LOWER BOUNDS FOR THE EIGENVALUES OF THE $\operatorname{Spin}^{c}$ DIRAC OPERATOR ON SUBMANIFOLDS
}

\author{
ROGER NAKAD AND JULIEN ROTH
}

\begin{abstract}
We prove lower bounds for the eigenvalues of the $\mathrm{Spin}^{\mathrm{c}}$ Dirac operator on submanifolds. These estimates are expressed in terms of extrinsic and intrinsic quantities. We also give estimates involving the Energy-Momentum tensor as well as conformal bounds. The limiting cases of these estimates give rise to particular spinor fields, called generalized twisted Killing spinors, which are also studied.
\end{abstract}

\section{INTRODUCTION AND PREMILINARIES}

The limiting cases of estimates for eigenvalues of the Dirac operator on compact (with or without boundary) manifolds give rise to examples of special geometries. For instance, equality in the classical inequalitiy of Friedrich [3]

$$
\lambda_{1} \leqslant \frac{n}{4(n-1)} \inf _{M} S_{c a l},
$$

where $n$ is the dimanesion of the manifold $M$ and $S c a l_{M}$ its scalar curvature, forces the manifold to be Einstein with positive scalar curvature, due to the fact that the eigenspinor associated with the first eigenvalue is then a real Killing spinor.

This is also the case for the conformal inequality of Hijazi [9]

$$
\lambda_{1} \leqslant \frac{n}{4(n-1)} \mu_{1},
$$

where $\mu_{1}$ is the first eigenvalue of the Yamabe operator as well for the inequality involving the Energy-momentum tensor [10]

$$
\lambda_{1} \leqslant \frac{1}{4} \inf _{M}\left(S c a l_{M}+\left|Q_{\varphi}\right|^{2}\right),
$$

where $Q_{\varphi}$ is the Energy-Momentum tensor associated with a first eigenspinor $\varphi$.

On the other hand, in the recent years, many estimates have been proved for the eigenvalues of the $\operatorname{Spin}^{c}$ Dirac operator. A great difference between both cases if that the equality cases for $\mathrm{Spin}^{\mathrm{c}}$ lower bounds are less and therefore give larger classes of limiting manifolds (see [8, 13, 14] for instance).

In this note, we prove a new lower bound for the eigenvalues of the $\operatorname{Spin}^{c}$ Dirac operator on submanifolds of $\operatorname{Spin}^{c}$ manifolds (see Theorem 2.1). This generalizes for the $\operatorname{Spin}^{c}$ case, previous estimates by Hijazi-Zhang [11, 12] and Ginoux-Morel [5]. The limiting case of this estimate is characterized by the existence of particular spinor fields called generalized twisted Killing spinors. We will study these particular spinor fields and show that under a natural assumption on the dimension and the codimension of the submanifold, they are in fact twisted Killing spinors which generalize naturally the usual Killing spinors for twisted spinor bundles.

Finally, we also prove conformal estimates (Theorem 2.3) as well as estimates involving the Energy-Momentum tensor associated with an eigenspinor (Theorem 2.2].

2010 Mathematics Subject Classification. 53C27, 53C42.

Key words and phrases. Submanifolds, Dirac Operator, Eigenvalues, Spin ${ }^{\mathrm{c}}$ spinors, Twisted Killing spinors. 
We recall briefly some basic facts about $\operatorname{Spin}^{c}$ manifolds and their hypersurfaces (the reader can refer to [1, 2, 4, 5]). Let $\left(\widetilde{M}^{m+n}, \widetilde{g}\right)$ be a Riemannian $\operatorname{Spin}^{\mathrm{c}}$ manifold and $M^{m}$ a submanifold isometrically immersed into $\widetilde{M}$. Assume that $M$ is also $\operatorname{Spin}^{\mathrm{c}}$ and denote by $N M$ the normal bundle of the immersion of $M$ into $\widetilde{M}$. We denote by $i \widetilde{\Omega}$ (resp. $i \Omega$ ) the curvature 2 -form of the corresponding auxiliary line bundle. Since the manifolds $M$ and $\widetilde{M}$ are $\operatorname{Spin}^{\mathrm{c}}$, there exists a $\operatorname{Spin}^{\mathrm{c}}$ structure on the bundle $N M$. We denote by $\Sigma N$ the $\operatorname{Spin}^{\mathrm{c}}$ bundle of $N M$ and let

$$
\Sigma:= \begin{cases}\Sigma M \otimes \Sigma N & \text { if } m \text { or } n \text { is even } \\ \Sigma M \otimes \Sigma N \oplus \Sigma M \otimes \Sigma N & \text { otherwise }\end{cases}
$$

It is well known that there is a natural isomorphism between $\Sigma$ and $\Sigma \widetilde{M}_{\mid M}$. Moreover, we denote by $\nabla$ the covariant derivative on $\Sigma$ defined by

$$
\nabla:= \begin{cases}\nabla^{\Sigma M} \otimes \mathrm{Id}+\mathrm{Id} \otimes \nabla^{\Sigma N} & \text { if } m \text { or } n \text { is even }, \\ \nabla^{\Sigma M} \otimes \mathrm{Id}+\mathrm{Id} \otimes \nabla^{\Sigma N} \oplus \nabla^{\Sigma M} \otimes \mathrm{Id}+\mathrm{Id} \otimes \nabla^{\Sigma N} & \text { otherwise. }\end{cases}
$$

We have the following identification between the Clifford multiplication

$$
X \cdot_{M} \varphi=\left.\left(X \cdot \omega_{\perp} \cdot \psi\right)\right|_{M},
$$

where $\varphi=\left.\psi\right|_{M}, \psi \in \Gamma(\Sigma \widetilde{M}), \omega_{\perp}:=\omega_{n}$ if $n$ is even and $\omega_{\perp}=-i \omega_{n}$ if $n$ is odd, with $\omega_{n}=i^{\left[\frac{n+1}{2}\right]} \nu_{1} \cdots \nu_{n}$ the complex volume element of the normal bundle. We also recall the $\operatorname{Spin}^{c}$ Gauss formula

$$
\widetilde{\nabla}_{X} \varphi=\nabla_{X} \varphi+\frac{1}{2} \sum_{j=1}^{m} e_{j} \cdot B\left(X, e_{j}\right) \cdot \varphi
$$

where $\widetilde{\nabla}$ is the spinorial connection on $\widetilde{M}$ and $B$ is the second fundamental form of $M$ in $\widetilde{M}$. We will denote by $H$ the mean curvature.

Now, let us define the following Dirac operators

$$
\mathbf{D}=\sum_{j=1}^{m} e_{j} \cdot \nabla_{e_{j}}, \quad \widetilde{D}:=\sum_{j=1}^{m} e_{j} \cdot \widetilde{\nabla}_{e_{j}},
$$

and

$$
D_{H}=(-1)^{n} \omega_{\perp} \cdot \widetilde{D}=(-1)^{n} \omega_{\perp} \cdot \mathbf{D}+\frac{1}{2} H \cdot \omega_{\perp} \cdot
$$

Clearly, from the spinorial Gauss formula (2), we have $\widetilde{D}=\mathbf{D}-\frac{1}{2} H$. Moreover (see [1, Lemma 2.1]), $\mathbf{D}$ and $D_{H}$ are formally self-adjoint and $D_{H}^{2}=\widetilde{D}^{*} \widetilde{D}$ where $\widetilde{D}^{*}$ is the formal adjoint of $\widetilde{D}$ with respect to the $L^{2}$-scalar product $\int_{M}(\cdot, \cdot) d v_{g}$.

We finish this section of preliminaries by the two following lemmas. The first one generalizes the classical Lichnerowicz formula in the context of twisted $\mathrm{Spin}^{c}$ spinor bundles. Before stating the lemma, we need to introduce the following function associated to a spinor field $\varphi \in \Gamma(\Sigma), R_{\varphi}^{N}:=2 \sum_{i, j=1}^{n}\left\langle e_{i} \cdot e_{j} \cdot I d \otimes \mathcal{R}_{e_{i}, e_{j}}^{N} \varphi, \frac{\varphi}{|\varphi|^{2}}\right\rangle$, on $M_{\varphi}=\{x \in M \mid \varphi(x) \neq 0\}$. Here, $\mathcal{R}_{e_{i}, e_{j}}^{N}$ stands for the spinorial curvature of the normal bundle $N M$.

Proposition 1.1 (Twisted Lichnerowicz formula). For any spinor field $\varphi \in \Gamma(\Sigma)$, pointwise on $M_{\varphi}$, we have

$$
\left\langle\mathbf{D}^{2} \varphi, \varphi\right\rangle=\left\langle\nabla^{*} \nabla \varphi, \varphi\right\rangle+\frac{1}{4}\left(\operatorname{Scal}_{M}+R_{\varphi}^{N}\right)|\varphi|^{2}+\frac{i}{2}\langle\Omega \cdot \varphi, \varphi\rangle .
$$


Proof: We give the proof in the case where $m$ or $n$ is even. The other case is similar. We compute the square of the Dirac operator $\mathbf{D}$ acting on $\varphi=\alpha \otimes \sigma$. We have

$$
\mathbf{D}^{2} \varphi=\nabla^{*} \nabla \varphi+\frac{1}{2} \sum_{i, j=1}^{m} e_{i} \cdot e_{j} \cdot \mathcal{R}_{e_{i}, e_{j}} \varphi,
$$

where $\nabla^{*}$ is the formal adjoint of $\nabla$ and $\mathcal{R}$ is the spinorial curvature associated with the connection $\nabla$. From the definition of $\nabla$, we see easily that

$$
\mathcal{R}_{e_{i}, e_{j}} \varphi=\left(\mathcal{R}_{e_{i}, e_{j}}^{M} \sigma\right) \otimes \alpha+\sigma \otimes\left(\mathcal{R}_{e_{i}, e_{j}}^{N} \alpha\right) .
$$

Then, a classical computation on each factor gives the desired formula.

We have this second elementary lemma

Proposition 1.2. For any spinor field $\varphi \in \Gamma(\Sigma)$, we have

$$
\langle i \Omega \cdot \varphi, \varphi\rangle \geq-\frac{c_{m}}{2}|\Omega||\varphi|^{2} .
$$

Proof: If $m$ or $n$ is even, then $\Sigma M \otimes \Sigma N$ and a spinor $\varphi \in \Sigma$ can be written $\varphi=\sigma \otimes \alpha$. Hence, we have

$$
\begin{aligned}
\langle i \Omega \cdot \varphi, \varphi\rangle & =\langle i \Omega \cdot(\sigma \otimes \alpha), \sigma \otimes \alpha\rangle=\left\langle i\left(\Omega_{M} \sigma\right) \otimes \alpha, \sigma \otimes \alpha\right\rangle \\
& =\left\langle i\left(\Omega_{\dot{M}}^{\dot{*}} \sigma\right), \sigma\right\rangle|\alpha|^{2} \geq-\frac{c_{m}}{2}|\Omega||\sigma|^{2}|\alpha|^{2} \geq-\frac{c_{m}}{2}|\Omega||\varphi|^{2} .
\end{aligned}
$$

Note that we use the fact that the scalar product on $\Sigma$ is the product of the scalar products on $\Sigma M$ and $\Sigma N$. We also use the classical estimate on $M$, that is $\langle i \Omega \cdot \sigma, \sigma\rangle \geq-\frac{c_{m}}{2}|\Omega||\sigma|^{2}$ (see [8]). If $m$ and $n$ are odd, then $\Sigma=\Sigma M \otimes \Sigma N \oplus \Sigma M \otimes \Sigma N$ and a spinor $\varphi \in \Sigma$ is of the form $\varphi=\left(\alpha \otimes \sigma, \sigma^{\prime} \otimes \alpha^{\prime}\right)$. Thus, we have

$$
\begin{aligned}
\langle i \Omega \cdot \varphi, \varphi\rangle & =\langle i \Omega \cdot(\sigma \otimes \alpha), \sigma \otimes \alpha\rangle+\left\langle i \Omega \cdot\left(\sigma^{\prime} \otimes \alpha^{\prime}\right), \sigma^{\prime} \otimes \alpha^{\prime}\right\rangle \\
& \geq-\frac{c_{m}}{2}|\Omega|\left(|\sigma|^{2}|\alpha|^{2}+\left|\sigma^{\prime}\right|^{2}\left|\alpha^{\prime}\right|^{2}\right) \geq-\frac{c_{m}}{2}|\Omega \| \varphi|^{2} .
\end{aligned}
$$

This concludes the proof.

\section{EIgENVALUE ESTIMATES FOR SUBMANIFOLDS}

Now, we have all the ingredients to state the eigenvalue estimates. We begin by the following basic estimates involving intrinsic terms (scalar curvature, curvature of the line bundle over $M$ ) and extrinsic terms (mean curvature and spinorial normal curvature). This result generalizes in the $\operatorname{Spin}^{c}$ setting the estimate of Hijazi-Zhang [11] (extending to any codimension by Ginoux-Morel [5]).

Theorem 2.1. Let $\left(M^{m}, g\right)$ be a compact Riemannian $\operatorname{Spin}^{\mathrm{c}}$ manifold isometrically immersed into a Riemannian $\operatorname{Spin}^{c}$ manifold $\left(\widetilde{M}^{m+n}, \widetilde{g}\right)$. Consider a non-trivial eigenspinor field $\varphi \in \Gamma(\Sigma)$ for the submanifold Dirac operator $D_{H}$, i.e. $D_{H} \varphi=\lambda \varphi$. Assume that $m \geq 2$ and

$$
\operatorname{Scal}_{M}+R_{\varphi}^{E}-c_{m}\left|\Omega^{M}\right|>\frac{m-1}{m}\|H\|^{2}>0
$$

on $M_{\varphi}$, then, we have

$$
\lambda^{2} \geq \frac{1}{4} \inf _{M_{\varphi}}\left(\sqrt{\frac{m}{m-1}\left(S_{c a l}+R_{\varphi}^{N}-c_{m}|\Omega|\right)}-\|H\|\right)^{2} .
$$

Moreover, if equality holds, then $\varphi$ is a twisted generalized Killing spinor. 
Proof: Let $\lambda$ be an eigenvalue of the submanifold Dirac operator $D_{H}$ and $q$ a smooth function, nowhere equal to $\frac{1}{m}$. We consider the following modified connection $\nabla^{\lambda, q}$ defined by

$$
\nabla_{X}^{\lambda, q} \psi=\nabla_{X} \psi+\frac{1-q}{2(1-m q)} X \cdot H \cdot \psi+q \lambda X \cdot \omega_{\perp} \cdot \psi
$$

for any spinor field $\psi \in \Gamma(\Sigma)$. Let $\varphi$ be an eigenspinor for $D_{H}$ associated with the eigenvalue $\lambda$. Using the Twisted Lichnerowicz formula (3), we can easily compute

$\int_{M}\left|\nabla^{\lambda, q} \varphi\right|^{2} \mathrm{v}_{g}=\int_{M}\left(1+m q^{2}-2 q\right)\left[\lambda^{2}-\frac{1}{4}\left(\frac{\operatorname{Scal}_{M}+R_{\varphi}^{N}}{1+m q^{2}-2 q}-\frac{(m-1)\|H\|^{2}}{(1-m q)^{2}}\right)|\varphi|^{2}\right.$

$$
\left.-\frac{i}{2\left(1+m q^{2}-2 q\right)}<\Omega \cdot \varphi, \varphi>\right] \mathrm{v}_{g}
$$

Then, using Inequality $(5)$ and by assuming $m\left(\operatorname{Scal}_{M}+R_{\varphi}^{N}-c_{m}|\Omega|\right)>(m-1)\|H\|^{2}>0$, we can choose $q$ so that

$$
(1-m q)^{2}=\frac{(m-1)\|H\|}{\sqrt{\frac{m}{m-1}\left(\mathrm{Scal}_{M}+R_{\varphi}^{N}-c_{m}|\Omega|\right)}-\|H\|},
$$

on $M_{\varphi}$. Inserting (7) in (6), and since the complement of $M_{\psi}$ in $M$ is of measure 0 , we conclude because the left member of (6) is nonnegative.

If equality occurs, $\nabla_{X}^{\lambda, q} \varphi=0$, which implies that $|\varphi|$ is constant on $M$ and

$$
\mathbf{D} \varphi=\frac{m(1-q)}{2(1-m q)} H \cdot \varphi+m q \lambda \omega_{\perp} \cdot \varphi
$$

On the other hand, since $\varphi$ is an eigenspinor for $D_{H}$ and the link between $D_{H}$ and $\mathbf{D}$, we get

$$
\begin{aligned}
0 & =\lambda \omega_{\perp} \cdot \varphi+\frac{H}{2} \cdot \varphi-\frac{m(1-q)}{2(1-m q)} H \cdot \varphi-m q \lambda \omega_{\perp} \cdot \varphi \\
& =(1-m q)^{2} \lambda \omega_{\perp} \cdot \varphi-(m-1) \frac{H}{2} \cdot \varphi
\end{aligned}
$$

Moreover, equality also implies $2|\lambda|=\sqrt{\frac{m}{m-1}\left(\operatorname{Scal}_{M}+R_{\varphi}^{N}-c_{m}|\Omega|\right)}-\|H\|$. From the expression of $q$ and the above relation, we have $\omega_{\perp} \cdot \varphi=\operatorname{sgn}(\lambda) \frac{H}{\|H\|} \cdot \varphi$ and thus $\varphi$ satisfies $\nabla_{X} \varphi=-\frac{f}{m} X \cdot \omega_{\perp} \cdot \varphi$, with $f=\frac{\operatorname{sgn}(\lambda)}{2} \sqrt{\frac{m}{m-1}\left(\operatorname{Scal}_{M}+R_{\varphi}^{N}-c_{m}|\Omega|\right)}$. That is, $\psi$ is a generalized twisted Killing spinor. Note that here $f$ is a priori a function. We will see in Section 3 that under some assumptions on the dimensions $m$ and $n$, the function $f$ is constant.

Now, we define the Energy-Momentum tensor associated with a spinor field $\psi \in \Gamma(\Sigma)$ on $M_{\psi}$ by

Note that

$$
Q_{i j}^{\psi}=\frac{1}{2}\left(e_{i} \cdot \omega_{\perp} \cdot \nabla_{e_{j}}+e_{j} \cdot \omega_{\perp} \cdot \nabla_{e_{i}}, \frac{\psi}{|\psi|^{2}}\right)
$$

$$
Q_{i j}^{\psi}=\frac{1}{2}\left(e_{i} \cdot{ }_{M} \nabla_{e_{j}}+e_{j} \cdot M \nabla_{e_{i}}, \frac{\psi}{|\psi|^{2}}\right)
$$

so it is the intrinsic energy momentum tensor and it is the one appearing in the Einstein Dirac equation. We have the following estimate involving the Energy-Momentum tensor.

Theorem 2.2. Let $\left(M^{m}, g\right)$ be a compact Riemannian $\mathrm{Spin}^{\mathrm{c}}$ manifold isometrically immersed into a Riemannian $\operatorname{Spin}^{\mathrm{c}}$ manifold $\left(\widetilde{M}^{m+n}, \widetilde{g}\right)$. Consider a non-trivial eigenspinor field $\varphi \in \Gamma(\Sigma)$ for the submanifold Dirac operator $D_{H}$, i.e. $D_{H} \varphi=\lambda \varphi$. Assume that $m \geq 2$ and

$$
\operatorname{Scal}_{M}+R_{\varphi}^{N}+4\left|Q^{\varphi}\right|^{2}-c_{m}\left|\Omega^{M}\right|>\|H\|^{2}>0
$$


on $M_{\varphi}$, then, we have

$$
\left.\lambda^{2} \geq \frac{1}{4} \inf _{M_{\varphi}}\left(\operatorname{Scal}_{M}+R_{\varphi}^{N}+4\left|Q^{\varphi}\right|^{2}-c_{m}|\Omega|\right)-\|H\|\right)^{2} .
$$

Moreover, if equality holds, then $\varphi$ is a twisted (symmetric) EM-spinor.

Proof: For any real function $q$ that never vanishes, consider the modified covariant derivative defined on $\Gamma(\Sigma)$ by

$$
\nabla_{e_{i}}^{Q} \psi=\nabla_{e_{i}} \psi-\frac{1}{2 m q} e_{i} \cdot H \cdot \psi+(-1)^{n+1} q \lambda e_{i} \cdot \omega_{\perp} \cdot \psi+\sum_{j} Q_{i j}^{\psi} e_{j} \cdot \omega_{\perp} \cdot \psi
$$

Again, we can compute, for an eigenspinor $\varphi$

$$
\begin{aligned}
\int_{M}\left|\nabla^{Q} \varphi\right|^{2} \mathrm{v}_{g}= & \int_{M}(1+m q)^{2}\left[\lambda^{2}-\frac{1}{4}\left(\frac{\mathrm{Scal}_{M}+R_{\varphi}^{N}+4\left|Q^{\varphi}\right|^{2}}{\left(1+m q^{2}\right)}-\frac{\|H\|^{2}}{m q^{2}}\right)\right]|\varphi|^{2} \mathrm{v}_{g} \\
& -\frac{1}{4} \int_{M}\left(1+m q^{2}\right)\left[\frac{2}{m q\left(1+m q^{2}\right)}\left(\|H\|^{2}-\frac{\left.<H \cdot \varphi, \omega_{\perp} \cdot \varphi\right\rangle^{2}}{|\varphi|^{4}}\right)\right]|\psi|^{2} \mathrm{v}_{g} \\
\text { (8) } & -\frac{i}{2} \int_{M}<\Omega \cdot \varphi, \varphi>\mathrm{v}_{g}
\end{aligned}
$$

Now, we use again $(5)$ and if moreover, $\operatorname{Scal}_{M}+R_{\varphi}^{N}-c_{M}|\Omega|+4\left|Q^{\varphi}\right|^{2}>\|H\|^{2}>0$, we take

$$
q=\sqrt{\frac{\|H\|}{m\left(\sqrt{\mathrm{Scal}_{M}+R_{\varphi}^{N}-c_{M}|\Omega|+4\left|Q^{\varphi}\right|^{2}}-\|H\|\right)}}
$$

and then by the Cauchy Schwarz inequality, we have

$$
\|H\|^{2}-\frac{<H \cdot \varphi, \omega_{\perp} \cdot \varphi>^{2}}{|\varphi|^{4}} \geq 0 .
$$

If equality holds, then $\nabla^{Q} \varphi=0$ and equality occurs in the Cauchy-Schwarz inequality, that is, $\|H\|^{2}-\frac{\left.<H \cdot \varphi, \omega_{\perp} \cdot \varphi\right\rangle^{2}}{|\varphi|^{4}}=0$. Thus, proceeding as in the proof of Theorem 2.1. we deduce that $\nabla_{X} \varphi=-Q(X) \cdot \omega_{\perp} \cdot \varphi$, that is $\psi$ is a twisted (symmetric) Energy-Momentum spinor (EM-spinor).

Note that, by a completely similar computation, we can obtain a lower bound involving both symmetric and skew-symmetric Energy-Momentum tensors as in [7]. We do not write it in this note.

Finally, following the idea of Hijazi [9], we consider a conformal change of the metrc $\bar{g}=e^{2 u} \widetilde{g}$. Let $\Sigma \longrightarrow \bar{\Sigma}, \psi \longrightarrow \bar{\psi}$ be the corresponding isometry between the two spinor bundles. Recall that for 2 spinors $\psi$ and $\varphi$ on $\Sigma$ and for any vector field $X$ on $\widetilde{M}$, we have

Note that we have

$$
(\varphi, \psi)=(\bar{\varphi}, \bar{\psi})_{\bar{g}} \quad \text { and } \quad \bar{X}^{-} \cdot \bar{\psi}=\overline{X \cdot \psi}
$$

$$
\overline{\mathbf{D}}\left(e^{\frac{-(m-1)}{2} u} \bar{\psi}\right)=e^{\frac{-(m+1)}{2} u} \overline{\mathbf{D} \psi}
$$

where $\overline{\mathbf{D}}$ denotes the Dirac operator w.r.t the metric $\bar{g}$. Moreover, the corresponding mean curvature is given by

$$
\bar{H}=e^{-2 u}\left(H-m \operatorname{grad}^{N} u\right)
$$

Now, assume that $\operatorname{grad}^{N} u=0$, then $D_{H}$ is also conformally covariant and we have

$$
\overline{D_{H}}\left(e^{\frac{-(m-1)}{2} u} \bar{\psi}\right)=e^{\frac{-(m+1)}{2} u} \overline{D_{H}} \psi
$$

From now on, we will consider regular conformal change of the metric $g$, i.e., $\operatorname{grad}^{N} u=0$. 
Theorem 2.3. Let $\left(M^{m}, g\right)$ be a compact Riemannian $\mathrm{Spin}^{\mathrm{c}}$ manifold isometrically immersed into a Riemannian Spin ${ }^{c}$ manifold $\left(\widetilde{M}^{m+n}, \widetilde{g}\right)$. Consider a non-trivial eigenspinor field $\varphi \in \Gamma(\Sigma)$ for the submanifold Dirac operator $D_{H}$, i.e. $D_{H} \psi=\lambda \psi$. For any regular conformal change of metric $\bar{g}=e^{2 u} \widetilde{g}$, assume that $m \geq 3$ and

$$
\overline{\mathrm{Scal}}_{M} e^{2 u}+R_{\varphi}^{N}+4\left|Q^{\psi}\right|^{2}-c_{m}\left|\Omega^{M}\right|>\|H\|^{2}>0
$$

on $M_{\varphi}$, then, we have

$$
\left.\lambda^{2} \geq \frac{1}{4} \inf _{M_{\psi}}\left(\sqrt{\overline{\mathrm{Scal}}_{M} e^{2 u}+R_{\psi}^{N}+4\left|Q^{\psi}\right|^{2}-c_{m}|\Omega|}\right)-\|H\|\right)^{2} .
$$

Proof: For $\psi \in \Gamma(\Sigma)$ an eigenspinor of $D_{H}$ with eigenvalue $\lambda$, let $\bar{\varphi}:=e^{\frac{-(n-1) u}{2}} \bar{\psi}$. then, we have $\overline{D_{H}} \bar{\varphi}=\lambda e^{-u} \bar{\varphi}$. Recall that

$$
\bar{\nabla}_{e_{i}} \bar{\psi}=\overline{\nabla_{e_{i}} \psi}-\frac{1}{2} \overline{e_{i} \cdot d u \cdot \psi}-\frac{1}{2} e_{i}(u) \bar{\psi}
$$

and $\overline{e_{i}}=e^{-u} e_{i}$. Now, it is straightforward to get $\bar{Q}_{\bar{i}, \bar{j}}^{\bar{\varphi}}=e^{-u} Q_{i, j}^{\psi}$, hence $\left|\bar{Q}^{\bar{\varphi}}\right|^{2}=$ $e^{-2 u}\left|Q^{\psi}\right|^{2}$. Equation 8 is also true on $(\widetilde{M}, \bar{g})$. If, we apply it to $\bar{\varphi}$, we get

$$
\begin{aligned}
\int_{M}\left|\nabla^{\bar{Q}} \bar{\varphi}\right|^{2} \mathrm{v}_{\bar{g}}= & \int_{M}(1+m q)^{2}\left[\left(\lambda e^{-u}\right)^{2}-\frac{1}{4}\left(\frac{\overline{\mathrm{Scal}}_{M}+\bar{R}_{\bar{\varphi}}^{N}+4\left|\bar{Q}^{\bar{\varphi}}\right|^{2}}{\left(1+m q^{2}\right)}-\frac{\|\tilde{H}\| \frac{2}{g}}{m q^{2}}\right)\right]|\bar{\varphi}|_{\bar{g}}^{2} \mathrm{v} \bar{g} \\
& -\frac{1}{4} \int_{M}\left(1+m q^{2}\right)\left[\frac{2}{m q\left(1+m q^{2}\right)}\left(\|\tilde{H}\|_{\bar{g}}^{2}-\frac{<\tilde{H} \cdot \bar{\varphi}, \bar{\omega}_{\perp} \cdot \bar{\varphi}>\frac{2}{g}}{|\varphi| \frac{4}{g}}\right)\right]|\bar{\varphi}| \frac{2}{g} \mathrm{v}_{\bar{g}} \\
\text { (9) } & -\frac{i}{2} \int_{M}<\Omega \cdot \bar{\varphi}, \bar{\varphi}>_{\bar{g}} \mathrm{v}_{\bar{g}}
\end{aligned}
$$

since $\tilde{H}=e^{-u} \bar{H}$ and $\bar{R}_{\bar{\varphi}}^{N}=e^{-2 u} R_{\psi}^{N}$, we have

$$
\begin{aligned}
\int_{M}\left|\nabla^{\bar{Q}} \bar{\varphi}\right|^{2} \mathrm{v}_{\bar{g}}= & \int_{M}(1+m q)^{2} e^{-2 u}\left[(\lambda)^{2}-\frac{1}{4}\left(\frac{\overline{\mathrm{Scal}}_{M} e^{2 u}+R_{\psi}^{N}+4\left|Q^{\psi}\right|^{2}}{\left(1+m q^{2}\right)}-\frac{\|H\|^{2}}{m q^{2}}\right)\right]|\bar{\varphi}|^{2} \mathrm{v}_{\bar{g}} \\
& -\frac{1}{4} \int_{M}\left(1+m q^{2}\right) e^{-2 u}\left[\frac{2}{m q\left(1+m q^{2}\right)}\left(\|H\|^{2}-\frac{<H \cdot \psi, \omega_{\perp} \cdot \psi>^{2}}{|\psi|^{4}}\right)\right]|\bar{\varphi}|^{2} \mathrm{v}_{\bar{g}} \\
(10) \quad & -\frac{i}{2} \int_{M}<\Omega \cdot \bar{\varphi}, \bar{\varphi}>_{\bar{g}} \mathrm{v}_{\bar{g}}
\end{aligned}
$$

Now, we use again $(5)$ and if moreover, $\overline{\mathrm{Scal}}_{M} e^{2 u}+R_{\psi}^{N}-c_{M}|\Omega|+4\left|Q^{\psi}\right|^{2}>\|H\|^{2}>0$, we take

$$
q=\sqrt{\frac{\|H\|}{m\left(\sqrt{\overline{\mathrm{Scal}}_{M} e^{2 u}+R_{\psi}^{N}-c_{M}|\Omega|+4\left|Q^{\psi}\right|^{2}}-\|H\|\right)}},
$$

and then we use the Cauchy Schwarz inequality

$$
\|H\|^{2}-\frac{<H \cdot \psi, \omega_{\perp} \cdot \psi>^{2}}{|\psi|^{4}} \geq 0,
$$

to get the desired result.

By taking $u$ as first eigenfunction of the Yamabe operator on $M$, we get the following corollary, where $\mu_{1}$ is the first eigenvalue of the Yamabe operator. 
Corollary 2.4. Under the assunptions of Theorem 2.3 and if

$$
\mu_{1}+R_{\psi}^{N}+4\left|Q^{\psi}\right|^{2}-c_{m}\left|\Omega^{M}\right|>\|H\|^{2}>0
$$

on $M_{\varphi}$, then, we have

$$
\left.\lambda^{2} \geq \frac{1}{4} \inf _{M_{\psi}}\left(\sqrt{\mu_{1}+R_{\psi}^{N}+4\left|Q^{\psi}\right|^{2}-c_{m}|\Omega|}\right)-\|H\|\right)^{2} .
$$

\section{Generalized twisted KiLling SPINORS}

We have seen in Theorem 2.1 that if equality occurs, then, the eigenspinor $\varphi$ is in fact a generalized twisted Killing spinor, that is satisfies the equation

$$
\nabla_{X} \varphi=f X \cdot \omega_{\perp} \cdot \varphi=f X_{\dot{M}} \varphi
$$

where $f$ is a real function. We prove the following

Proposition 3.1. Let $\left(M^{m}, g\right)$ be a compact Riemannian $\operatorname{Spin}^{\mathrm{c}}$ manifold isometrically immersed into a Riemannian $\operatorname{Spin}^{c}$ manifold $\left(\widetilde{M}^{m+n}, \widetilde{g}\right)$. Let $\varphi \in \Gamma(\Sigma)$ be a generalized twisted Killing spinor with real-valued function $f$. If $m>n+4$, then $f$ is constant.

Proof: We define the following forms for $p \in\{1, \cdots, m\}$,

$$
\omega_{p}\left(X_{1}, \cdots, X_{p}\right)=\left\langle\left(X_{1} \wedge X_{2} \wedge \cdots \wedge X_{p}\right)_{\dot{M}} \varphi, \varphi\right\rangle,
$$

We have the following easy facts (see [6, 8] for instance). For any $k \geqslant 0$, the forms $\omega_{4 k+1}$ and $\omega_{4 k+2}$ are imaginary-valued whereas the forms $\omega_{4 k+3}$ and $\omega_{4 k}$ are real-valued. Moreover, we have for any $p \geqslant 0$

$$
\mathrm{d} \omega_{p}=\left((-1)^{p} f-f\right) \omega_{p+1} .
$$

In particular, we have for any $k \geqslant 1$

$$
\mathrm{d} f \wedge \omega_{2 k}=0 .
$$

Assume that $f$ is not constant and let $x \in M$ such that $\mathrm{d} f \neq 0$, on a neighborhood $V$ of $x$. Hence $\mathrm{d} f^{\perp}$ is of dimension $m-1$ and we consider $\left\{e_{1}, \cdots, e_{m-1}\right\}$ an orthonormal frame of $\mathrm{d} f^{\perp}$. From this, we have

$$
\omega_{2 k}\left(e_{i_{1}}, \cdots, e_{i_{2 k}}\right)=0,
$$

for any subset $\left\{i_{1}, \cdots, i_{2 k}\right\}$ of $\{1, \cdots, m-1\}$. Thus, for $l=\left[\frac{m-1}{2}\right]$, we deduce that the spinor fields $\varphi, e_{i_{1}} \dot{M} e_{i_{2}} \dot{M} \varphi, \cdots$ and $e_{i_{1}} \dot{M}_{M} e_{i_{2}} \cdots e_{i_{2 l}} \dot{M} \varphi$ are orthonormal on $V$. Consequently, the space spanned by these spinors is a vector subspace of $\Sigma_{x}$ of complex dimension

$$
1+\left(\begin{array}{c}
m-1 \\
2
\end{array}\right)+\left(\begin{array}{c}
m-1 \\
4
\end{array}\right)+\cdots+\left(\begin{array}{c}
m-1 \\
2 l
\end{array}\right)=2^{m-2} .
$$

Since the complex dimension of $\Sigma$ is

$$
d(m, n)= \begin{cases}2^{\frac{m+n}{2}} & \text { if } m+n \text { is even } \\ 2^{\frac{m+n-1}{2}} & \text { if } m+n \text { is odd }\end{cases}
$$

we conclude that $2^{m-2} \leqslant d(m, n)$. Therefore, $f$ is constant if $2^{m-2}>d(m, n)$ which corresponds to the condition expressed in the statement of the Proposition. Indeed, on one hand, if $m+n$ is even, then $2^{m-2}>d(m, n)$ is equivalent to $m-2>\frac{n+m}{2}$, that is $m>n+4$. On the other hand, if $\mathrm{f} m+n$ is odd, then $2^{2 m-2}>d(m, n)$ is equivalent to $m-2>\frac{n+m-1}{2}$, that is $m>n+3$. But since $m+n$ is odd, then $m$ cannot be equal to $n+4$. hence we also have $m>n+4$.

Hence, we deduce the following 
Corollary 3.2. Let $\left(M^{m}, g\right)$ be a compact Riemannian $\mathrm{Spin}^{\mathrm{c}}$ manifold isometrically immersed into a Riemannian $\operatorname{Spin}^{\mathrm{c}}$ manifold $\left(\widetilde{M}^{m+n}, \widetilde{g}\right)$. if $m>n+4$ and equality occurs in Theorem 2.1 then $M$ admits a twisted Killing spinor.

Acknowledgment. The first author is indebted to the Center for Advanced Mathematical Sciences (CAMS, Lebanon) and the University of Paris-Est, Marne-la-Vallée for their hospitality and support.

\section{REFERENCES}

[1] C. Bär, Extrinsic bounds for eigenvalues of the Dirac operator, Ann. Glob. Anal. Geom. 16 (1998) 573-596.

[2] J. P. Bourguignon, O. Hijazi, J. L. Milhorat, A. Moroianu \& S. Moroianu, A spinorial approach to Riemannian and conformal geometry, Monograph in Mathematics, EMS.

[3] T. Friedrich, Der erste Eigenwert des Dirac-operators einer kompakten Riemannschen Mannigfaltigkeit nichtnegativer Skalarkrümmung, Math. Nach. 97 (1980), 117-146.

[4] T. Friedrich, Dirac operators in Riemannian Geometry, Graduate studies in mathematics, Volume 25, Americain Mathematical Society.

[5] N. Ginoux \& B. Morel, On eigenvalue estimates for the submanifold Dirac operator, Int. J. Math. 13 (2002), No. 5, 533-548.

[6] N. Grosse \& R. Nakad, Complex generalized Killing spinors on Riemannian Spin ${ }^{c}$ manifolds, arxiv:1311.0969 (to appear in Results in Mathematics).

[7] G. Habib, Energy-Momentum tensor on foliations, J. Geom. Phys. 57 (2007), 2234-2248.

[8] M. Herzlich et \& Moroianu, Generalized Killing spinors and conformal eigenvalue estimates for Spinc manifold, Ann. Glob. Anal. Geom. 17 (1999), 341-370.

[9] O. Hijazi, A conformal Lower Bound for the Smallest Eigenvalue of the Dirac Operator and Killing Spinors, Commun. Math. Phys. 104, (1986) 151-162.

[10] O. Hijazi, Lower bounds for the eigenvalues of the Dirac operator, J. Geom. Phys., 16 (1995) 27-38.

[11] O. Hijazi \& X. Zhang, Lower bounds for the Eigenvalues of the Dirac Operator, Part I. The Hypersurface Dirac Operator, Ann. Global Anal. Geom. 19, (2001) 355-376.

[12] O. Hijazi \& X. Zhang, Lower bounds for the Eigenvalues of the Dirac Operator, Part II. The Submanifold Dirac Operator, Ann. Global Anal. Geom. 19, (2001) 163-181.

[13] R. Nakad, Lower bounds for the eigenvalues of the Dirac operator on Spin ${ }^{\mathrm{C}}$ manifolds, J. Geom. Phys. 60 (2010), 1634-1642.

[14] R. Nakad \& J. Roth, The $\operatorname{Spin}^{c}$ Dirac operator on hypersurfaces and applications , Diff. Geom. Appl., 31 (1), (2013), pp 93-103

(J. Roth) LAMA, Université Paris-Est Marne-La-Vallée, Cité Descartes, Champs sur Marne, 77454 MARne-LA-VALLÉE CEDEX 2, FRANCE

E-mail address: julien.roth@u-pem. fr

(R. Nakad) Notre Dame University-Louaize, Faculty of Natural and Applied Sciences, De-

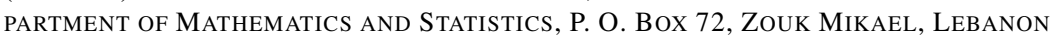

E-mail address: rnakad@ndu.edu. 1 b 